\title{
Water Wave Optimization Algorithm for Solving Economic Dispatch Problems with Generator Constraints
}

\author{
M. Siva ${ }^{1 *}$, R. Balamurugan ${ }^{1}$, L. Lakshminarasimman ${ }^{1}$ \\ ${ }^{1}$ Department of Electrical Engineering, Annamalai University, Annamalainagar, Tamilnadu, India \\ *Corresponding author's Email: vasi.siva@gmail.com
}

\begin{abstract}
This paper presents the application of Water Wave Optimization Algorithm (WWOA) for solving economic dispatch problems including practical generator constraints. WWOA is inspired by the shallow water wave theory. The efficiency of the WWO Algorithm for solving economic dispatch problems is demonstrated by implementing it on three test systems having three, six and fifteen generating units with non-linear characteristics of the generator such as ramp-rate limits, prohibited operating zones including the system transmission losses. The results of the proposed approach have been compared with existing results obtained by other solution techniques. The test results reveal the capability of the proposed algorithm as an effective tool for solving various economic dispatch problems in a power system.
\end{abstract}

Keywords: Water Wave Optimization Algorithm (WWOA); Economic load dispatch; ramp-rate constraints; prohibited operating zones; transmission losses; cost minimization.

\section{Introduction}

The economic load dispatch (ELD) is an essential and fundamental element in the optimal operation and control of modern power systems. Though the foremost objective of the economic dispatch problem is to reduce the total operating cost with the best combination of power outputs satisfying the total load demand and losses, many constraints like valve-point loadings, ramp-rate limit, prohibited operating zones etc., make the optimization problem highly nonlinear especially for larger power systems [1].

Some physical limitations, such as faults in machines or in its associated auxiliaries (boiler, feed pumps), make the units to have certain regions prohibited for operation termed as prohibited operating zones (POZs). The generators are not allowed to operate in these regions as they may experience increased vibrations in their shaft bearings that have to be prevented in real-time operation. Also due to unit generation output change restrictions, the real-time operating units can have ramp-rate limits [2].

The emphasis on direct search and stochastic methods is due to the observation that mathematical programming approaches are often not suitable for tackling such problems due to the non-convexity of the search space.

In traditional methods like lambda-iteration, gradient, base point method etc., the cost function is modelled as a single quadratic function but in practice the operating conditions of various generators are different and the input-output characteristics is remodelled as a piecewise quadratic function in the thermal power plants [3]. Even though the dynamic programming has no restrictions on the characteristics of the cost curves, they cause the dimension of the problem to be high which in turn requires more computational efforts to solve the problem [4]. For the past several years several AI techniques such as genetic algorithm, evolutionary programming $[5,6]$, differential evolution $[7,8,9]$, particle swarm optimization [10, $11,12,20]$, biogeography based optimization [13, 14], artificial bee colony algorithm $[15,16]$, 
exchange market algorithm [18] are employed to find the optimal solution for the economic dispatch problems.

Although there are several methods available for the economic dispatch problem, the larger the system, greater is the complexity which necessitates developing efficient algorithms to stably find an optimal solution. In this context, the focus of this work is to demonstrate the efficiency of a nature inspired approach for solving ELD problems of varying complexity.

In the first, the water wave theory was related to gravitational force and other forces dating back to Newton's work in 1687 [20] and later by the development of mathematical models like Laplace. Lagrange, Poisson made the linear wave theory advanced along with non-linear waves as considered by Stokes, Gerstner and Kelland [21].

Recently, a new metaheuristic optimization technique called Water Wave Optimization Algorithm (WWOA) has been proposed by Zheng [17] which is inspired by the shallow water wave models. Here, the idea is from the wave motions, which is controlled by the wave-current-bottom interactions and the wave turbulence theory [21, 22] in the search mechanism design for high dimensional optimization problems. The WWOA maintains the population of solutions, each of which is analogous to a "Wave" with a height ' $h$ ' and a wavelength ' $\lambda$ '. In this paper, the WWOA is used for obtaining the solutions for ELD problems with generator constraints. The major advantage of the proposed method is it's easy to implement, requires lesser number of population vectors, fewer control parameters and hence are very effective in search for the best solution in a high dimensional search space. To show the competitive nature and efficiency of WWOA, it is compared with some of the familiar metaheuristics techniques that were proposed in the recent years $[7,11,18,19]$.

The remaining of the paper is organized as follows: Section 2 describes different cases of economic dispatch problems. The three phases of WWO Algorithm and their mathematical model is presented in Section 3. Section 4 deals with the implementation part of the WWO Algorithm to economic dispatch problems. The results of the WWOA implementation to numerical test systems with $3,6,15$ units are compared with a few popular techniques with illustration and are presented in Section 5.

In order to emphasize the capability of the WWO Algorithm, the technique is applied to various convex and non-convex economic dispatch problems considering the existence of power system losses, ramp-rate limits and the prohibited operating zones.

\section{Problem formulation}

\subsection{Objective function}

The main objective of the ELD problem is to determine the optimal combination of power generations that minimizes the total generation cost satisfying the generator constraints. The traditional objective function of the ELD problem can be approximately represented as a single quadratic function.

\section{Minimizing the overall operational cost}

The overall operational cost is the sum of production cost and variable operation and maintenance cost. The objective function of minimizing the overall operational cost over the plan ning period is stated as,

$$
\operatorname{Min} F_{T}=\sum_{i=1}^{N_{g}} F_{i}\left(P_{G i}\right), \quad(\$ / h)
$$

Hence, the fitness function to be maximized is

$$
\operatorname{Max} F_{T}=-\left[\sum_{i=1}^{N g} F_{i}\left(P_{G i}\right)+h_{p p}\left[\left(P_{D}+P_{L}\right)-\sum_{i=1}^{N_{g}} P_{G i}\right]^{2}\right](\$ / h)
$$

where $F_{i}\left(P_{G i}\right)=a_{i}+b_{i} P_{G i}+c_{i} P_{G i}^{2},(\$ / h), \mathrm{i}=1,2, \ldots, \mathrm{N}_{\mathrm{g}}$. $F_{T}$ is the total generation cost $(\$ / \mathrm{h}) ; F_{i}$ is the cost function of the $\mathrm{i}^{\text {th }}$ generator; $a_{i}, b_{i}, c_{i}$ are the cost coefficients of the $\mathrm{i}^{\text {th }}$ generator, $P_{G i}$ is the power output (MW) of the $\mathrm{i}^{\text {th }}$ generator and $N_{g}$ is the number of generators, $h_{p p}$ is the penalty factor, $\mathrm{P}_{\mathrm{D}}$ is the total power demand (MW). $\mathrm{P}_{\mathrm{L}}$ is the total power loss (MW).

\subsection{Power balance constraints}

The total power generated should be equal to the total load demand $\left(\mathrm{P}_{\mathrm{D}}\right)$ along with the transmission line loss $\left(\mathrm{P}_{\mathrm{L}}\right)$ as given by,

$$
\sum_{i=1}^{N_{g}} P_{i}=P_{D}+P_{L}
$$

The loss can be calculated using B-loss coefficients by Kron's formula as,

$$
P_{L}=\sum_{i=1}^{N_{g}} \sum_{j=1}^{N_{g}} P_{i} \cdot B_{i j} \cdot P_{j}+\sum_{i=1}^{N_{g}} B_{0 i} \cdot P_{i}+B_{00}
$$

where $B_{i j}, B_{0 i}, B_{00}$ are the $\mathrm{B}$ coefficients. 


\subsection{Generator capacity constraints}

The generated power output $\left(P_{i}\right)$ of each generator should vary within its minimum $\left(P_{i, \min }\right)$ and maximum $\left(P_{i, \max }\right)$ limits. This inequality constraint is represented as,

$$
P_{i, \min } \leq P_{i} \leq P_{i, \max }
$$

\subsection{Ramp-rate limits}

The power generated by the $\mathrm{i}^{\text {th }}$ generator $p_{i}^{o}$ in certain interval $(\mathrm{t})$ may neither exceed the previous interval (t-1) generation $p_{i}^{o}$ by more than $\mathrm{UR}_{\mathrm{i}}$ (up-ramp rate limit) nor less than $\mathrm{DR}_{\mathrm{i}}$ (down-ramp rate limit). This constraint is represented as,

$\max \left(p_{i}^{\min }, p_{i}^{0}-D R_{i}\right) \leq p_{i} \leq \min \left(p_{i}^{\max }, p_{i}^{0}+U R_{i}\right)$

i.e., as generation increases or decreases, $\mathrm{p}_{\mathrm{i}}-p_{i}^{o} \leqslant U R i$ (or) $p_{i}^{o}-p i \leqslant D R i$ should be maintained respectively.

\subsection{Prohibited operating zones}

In practice, the machine operations are limited by boiler, feed pumps etc. The prohibited operating zones are the range of power output of a generator where the operation causes vibrations of the turbine shaft bearing caused by the opening and closing operations of the steam valve. The machines or their accessories may get damaged if they are made operate in these prohibited operating regions. To avoid these kinds of faults the generated power output should satisfy the POZ constraint. The permitted operating zones of generation is defined as,

$$
\left\{\begin{array}{l}
P_{i, \min } \leq P_{i} \leq P_{i, 1}^{l} \\
P_{i, k-1}^{u} \leq P_{i} \leq P_{i, k}^{l} \\
P_{i, z}^{u} \leq P_{i} \leq P_{i . \max }
\end{array}\right.
$$

where $p_{i, k}^{l}$ and $p_{i, k}^{u}$ are the lower and upper limits of $k^{t h}$ prohibited zone respectively. $z$ is the number of prohibited operating zones for the $\mathrm{i}^{\text {th }}$ generator.

\section{Water wave optimization algorithm}

\subsection{Inspiration}

Nature has been the main source of inspiration for the majority of the population-based stochastic optimization techniques which performs the optimiz ation randomly. The optimization process is usually started by creating a set of random solutions. These initial solutions are then combined, moved, or evolved over a predefined number of steps called iterations or generations. This is almost the main framework of all population-based algorithms. In this paper, the application of Water Wave Optimiz ation Algorithm (WWOA) is proposed to solve the economic dispatch problems with certain constraints. The WWO Algorithm was introduced by Zheng in the year 2015[17].

The WWOA has three important phases for finding solution to the problem at hand. They are wave propagation, breaking and refraction phase. In Wave propagation, the wave is propagated to a random position exactly once in an iteration. If a wave attains a lower sea depth (best fitness), it breaks into solitary waves which are formed in the breaking phase. Thus breaking is used for the intensive search (exploitation) in search spaces by producing random solitary waves around the current best position. While in the Refraction phase, the algorithm explores the search space for any other best solution and avoids search inactiveness (stagnation). Overall, these three phases plays a vital role in the finding optimal or near optimal solution for the problem. Here, each solution is represented as a 'wave' with corresponding height (h) and wavelength $(\lambda)$.

\subsection{Mathematical model of WWO Algorithm}

In prelude to the proposed WWO Algorithm (WWOA), the mathematical model of propagation, breaking and refraction is provided first.

\subsubsection{Propagation}

From the wave population, each wave is allowed to propagate only once in each iteration. Here the propagation operator shifts the original wave $x$ in each dimension to produce a new propagated wave $x^{\prime}$. The new wave is modelled by the following equation:

$$
x^{\prime}(d)=x(d)+r n d(-1,1) \cdot \lambda \cdot L(d)
$$

where $\operatorname{rnd}(-1,1)$ is a uniformly distributed random number within the range $[-1,1]$ and $L(d)$ is the length of the $\mathrm{d}^{\text {th }}$ dimension. $\lambda$ is the wavelength of wave $x$, which is updated after each generation, as follows:

$$
\lambda=\lambda \cdot \alpha^{-\left(f(x)-f_{\min }+\epsilon\right) /\left(f_{\max }-f_{\min +\epsilon)}\right.}
$$

where $\alpha$ is the wavelength reduction coefficient, where $\mathrm{f}(\mathrm{x})$ is the fitness of the original wave, $f_{\max }$ and $f_{\min }$ are respectively the maximum and minimum fitness values among the current population and $\epsilon$ is a very small positive number to avoid division-by-zero. The Eq. (9) ensures that the waves 
with higher fitness value have lower wavelengths and thus propagate with smaller ranges.

\subsubsection{Breaking}

In WWOA, the breaking operation is performed only on a wave $x$ that finds a new best solution (i.e., $x$ becomes the new $\left.x^{*}\right)$ and conduct a local search around $x^{*}$ using ' $k$ ' solitary waves to simulate wave breaking using Eq. (10).

$$
x^{\prime}(d)=x(d)+N(0,1) \cdot \beta \cdot L(d)
$$

where $\beta$ is the breaking coefficient. $N$ is the Gaussian random number, $L(d)$ is the length of the $\mathrm{d}^{\text {th }}$ dimension. If none of the solitary waves are better than $x^{*}, x^{*}$ is retained; otherwise $x^{*}$ is replaced by the fittest one among the solitary waves. Totally $k$ number of solitary waves $x^{\prime}$ are generated at each dimension $d$ and the value of $k$ is generated randomly between 1 and $k_{\max }$.

\subsubsection{Refraction}

During wave propagation, if the wave path is not perpendicular to the isobaths the wave gets deflected and the wave converges in shallow regions and diverges in deep regions. In WWOA, refraction is performed on the waves whose height decreases to zero. The position of the wave after refraction is calculated as,

$$
x^{\prime}(d)=N\left(\frac{x^{*}(d)+x(d)}{2}, \frac{\left|x^{*}(d)-x(d)\right|}{2}\right)
$$

where $N$ is a Gaussian random number, $x^{*}$ is the best solution found so far and $\mathrm{d}$ is the dimension of the problem. So the new position of the wave is a random number midway between the original and the current best known position. Once the refraction phase is ended, the wave height of $x^{\prime}$ is reset to its maximum value $h_{\max }$ and its wavelength is set by,

$$
\lambda^{\prime}=\lambda \frac{f(x)}{f\left(x^{\prime}\right)}
$$

\section{Implementation of WWO Algorithm for ELD problem}

There are four main control parameters in WWO Algorithm apart from the population size. They are: the maximum wave height $h_{\max }$, the wavelength reduction coefficient $\alpha$, the breaking coefficient $\beta$, and the maximum number $k_{\max }$ of breaking directions. In all our test system the parameters, $\alpha=$ $1.01, \beta=0.001$, and $h_{\max }=6$ are used for the study of economic dispatch problems and the maximum number of iterations is considered as the stopping criteria. The following are the parameter selection range as recommended by Zheng [17] in his literature.

\subsection{Parameter selection range}

Wavelength reduction coeff. $(\alpha)=(1.001$ to 1.01$)$

Breaking coefficient $(\beta) \quad=(0.001$ to 0.01$)$

Maximum wave height $\left(h_{\max }\right)=5$ or 6

Initial Wavelength $(\lambda) \quad=0.5$

Max. No. of breaking direction $\mathrm{k}_{\max }=\min (12, \mathrm{D} / 2)$ where, $\mathrm{D}$ is the problem dimension.

The flow chart of the proposed WWO Algorithm implemented to solve the economic dispatch problem is shown in figure 1.

\section{Numerical simulation results and discussion}

The WWO Algorithm was applied on three different test systems for investigating the optimization capability: The three test systems considered are (1) 3-unit system (2) 6-unit system and (3) 15-unit system. All three test systems are with ramp rate limits, prohibited operating zones and network losses. In order to verify the feasibility and efficiency performance of the proposed algorithm, it has been compared to other population based optimization techniques like, IDP [7], PSO [11], EMA [18] and GA [19]. When compared to the existing techniques, the proposed technique obtains quality solution with lesser number of iterations, minimum number of population vectors and fewer control parameters.

\subsection{Test system 1: 3 units system}

The system consists of three thermal units whose characteristics are given in table 1 and the data are taken from [18] that include transmission loss, ramp-rate limits and prohibited operating zones. The system load is set at $300 \mathrm{MW}$.

The kron's loss formula B-coefficients are taken from [19]. Here it is aimed to optimize or reduce the total fuel cost of the system including transmission losses and make it operate adapting the ramp-rate limits and prohibited operating zones.

The results obtained by the proposed algorithm on test system with three units and its comparison with EMA and GA are presented in table 2.

The convergence profile comparison of the cost function is depicted in figure 2. The comparison of generator power outputs by WWOA and other algorithms is shown in figure 3 . 


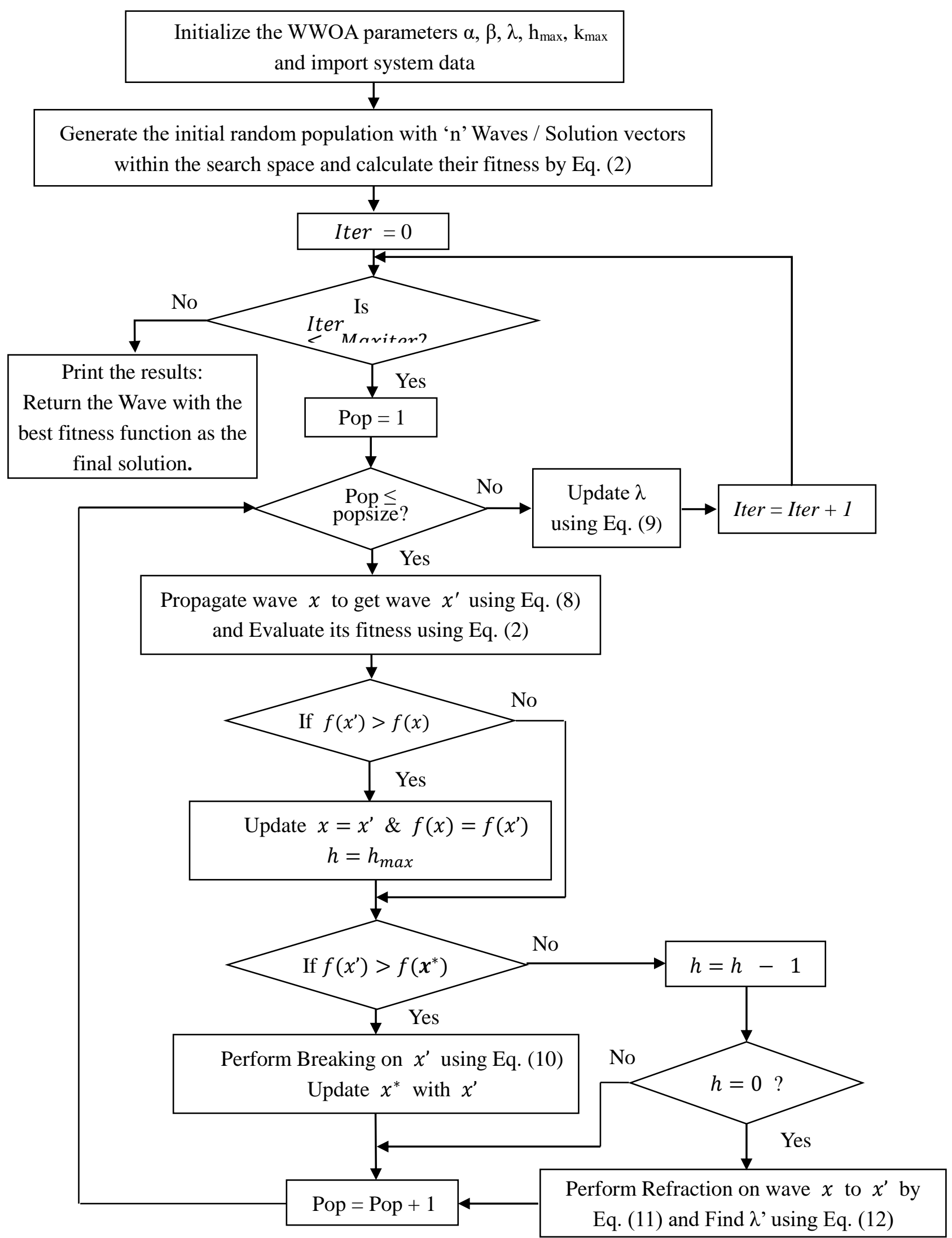

Figure.1 Flow chart of WWOA implementation for ELD problem 
Table 1. Operating characteristics data for 3, 6 and 15 unit systems

\begin{tabular}{|c|c|c|c|c|c|c|c|c|c|}
\hline Unit & $\begin{array}{l}P_{i, \min } \\
(\mathrm{MW})\end{array}$ & $\begin{array}{l}P_{i, \max } \\
(\mathrm{MW})\end{array}$ & $\begin{array}{c}a_{i} \\
(\$ / h)\end{array}$ & $\begin{array}{c}\boldsymbol{b}_{\boldsymbol{i}} \\
(\$ / \mathrm{MWh}) \\
\end{array}$ & $\begin{array}{c}c_{i} \\
\left(\$ / \mathbf{M W}^{2} / \mathbf{h}\right)\end{array}$ & $\begin{array}{c}U R_{i} \\
(\mathrm{MW}) \\
\end{array}$ & $\begin{array}{c}D R_{i} \\
(M W)\end{array}$ & $\begin{array}{c}P_{i}^{0} \\
(\mathbf{M W}) \\
\end{array}$ & $\begin{array}{c}\text { Prohibited Operating Zones } \\
(\mathrm{MW})\end{array}$ \\
\hline \multicolumn{10}{|c|}{3 units system } \\
\hline 1 & 50 & 250 & 328.13 & 8.663 & 0.00525 & 55 & 95 & 215 & {$[105,117] \quad[165,177]$} \\
\hline 2 & 5 & 150 & 136.91 & 10.04 & 0.00609 & 55 & 78 & 72 & {$[50,60] \quad\left[\begin{array}{ll}92 & 102\end{array}\right]$} \\
\hline 3 & 15 & 100 & 59.16 & 9.76 & 0.00592 & 45 & 64 & 98 & {$[25,32] \quad[60,67]$} \\
\hline \multicolumn{10}{|c|}{6 units system } \\
\hline 1 & 100 & 500 & 240 & 7 & 0.0070 & 80 & 120 & 440 & {$[210,240][350,380]$} \\
\hline 2 & 50 & 200 & 200 & 10 & 0.0095 & 50 & 90 & 170 & {$[90,110][140,160]$} \\
\hline 3 & 80 & 300 & 220 & 8.5 & 0.0090 & 65 & 100 & 200 & {$[150,170][210,240]$} \\
\hline 4 & 50 & 150 & 200 & 11 & 0.0090 & 50 & 90 & 150 & {$[80,90][110,120]$} \\
\hline 5 & 50 & 200 & 220 & 10.5 & 0.0080 & 50 & 90 & 190 & {$[90,110][140,150]$} \\
\hline 6 & 50 & 120 & 190 & 12 & 0.0075 & 50 & 90 & 110 & {$[75,85][100,108]$} \\
\hline \multicolumn{10}{|c|}{15 units system } \\
\hline 1 & 150 & 455 & 671 & 10.1 & 0.000299 & 80 & 120 & 400 & - \\
\hline 2 & 150 & 455 & 574 & 10.2 & 0.000183 & 80 & 120 & 300 & {$[185,225][305,335][420,450]$} \\
\hline 3 & 20 & 130 & 374 & 8.8 & 0.001126 & 130 & 130 & 105 & - \\
\hline 4 & 20 & 130 & 374 & 8.8 & 0.001126 & 130 & 130 & 100 & - \\
\hline 5 & 150 & 470 & 461 & 10.4 & 0.000205 & 80 & 120 & 90 & {$[180,200][305,335][390,420]$} \\
\hline 6 & 135 & 460 & 630 & 10.1 & 0.000301 & 80 & 120 & 400 & {$[230,255][365,395][430,455]$} \\
\hline 7 & 135 & 465 & 548 & 9.5 & 0.000364 & 80 & 120 & 350 & - \\
\hline 8 & 60 & 300 & 227 & 11.2 & 0.000338 & 65 & 100 & 95 & - \\
\hline 9 & 25 & 162 & 173 & 11.2 & 0.000807 & 60 & 100 & 105 & - \\
\hline 10 & 25 & 160 & 175 & 10.7 & 0.001203 & 60 & 100 & 110 & - \\
\hline 11 & 20 & 80 & 186 & 10.2 & 0.003586 & 80 & 80 & 60 & - \\
\hline 12 & 20 & 80 & 230 & 9.9 & 0.005513 & 80 & 80 & 40 & {$[30,40][55,65]$} \\
\hline 13 & 25 & 85 & 225 & 13.1 & 0.000371 & 80 & 80 & 30 & - \\
\hline 14 & 15 & 55 & 309 & 12.1 & 0.001929 & 55 & 55 & 20 & - \\
\hline 15 & 15 & 55 & 323 & 12.4 & 0.004447 & 55 & 55 & 20 & - \\
\hline
\end{tabular}

Table 2. Comparison of results for a three unit system $(\mathrm{Pd}=300 \mathrm{MW})$

\begin{tabular}{cccc}
\hline $\begin{array}{c}\text { Unit } \\
\text { (MW) }\end{array}$ & $\begin{array}{c}\text { WWOA } \\
\text { (Proposed) }\end{array}$ & EMA [18] & GA [18] \\
\hline $\mathrm{P} 1$ & 200.2231 & 200.5892 & 194.265 \\
$\mathrm{P} 2$ & 78.6042 & 78.2520 & 50.000 \\
$\mathrm{P} 3$ & 34.0000 & 34.0000 & 79.627 \\
$\sum \mathrm{P}_{\mathrm{Gi}}$ & 312.8274 & 312.8413 & 323.89 \\
$\mathrm{P}_{\text {Loss }}$ & 12.8274 & 12.8413 & 24.011 \\
$\mathrm{~T} . \mathrm{Gen}$. & 3634.7374 & 3634.7683 & 3737.20 \\
Cost $(\$ / \mathrm{h})$ & & & \\
\hline
\end{tabular}

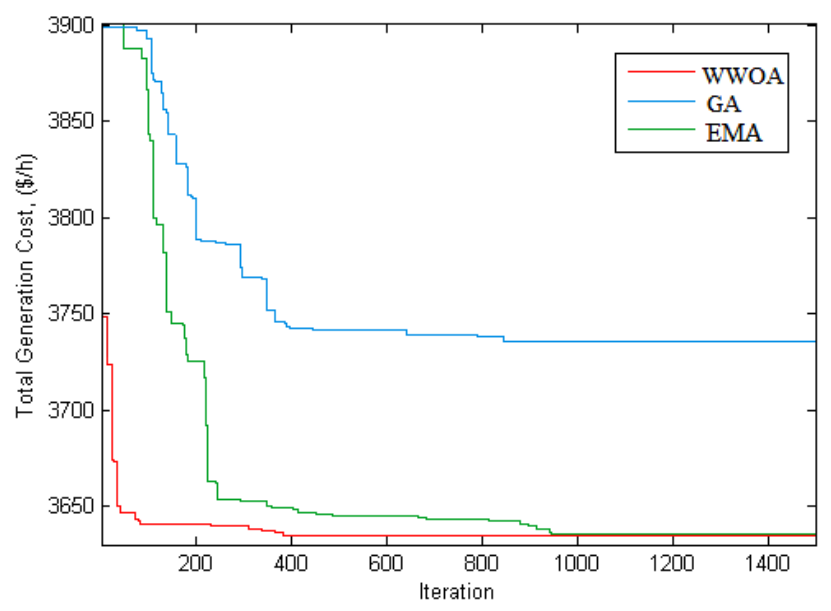

Figure. 2 Convergence characteristics comparison of WWOA with other methods for a three unit system 


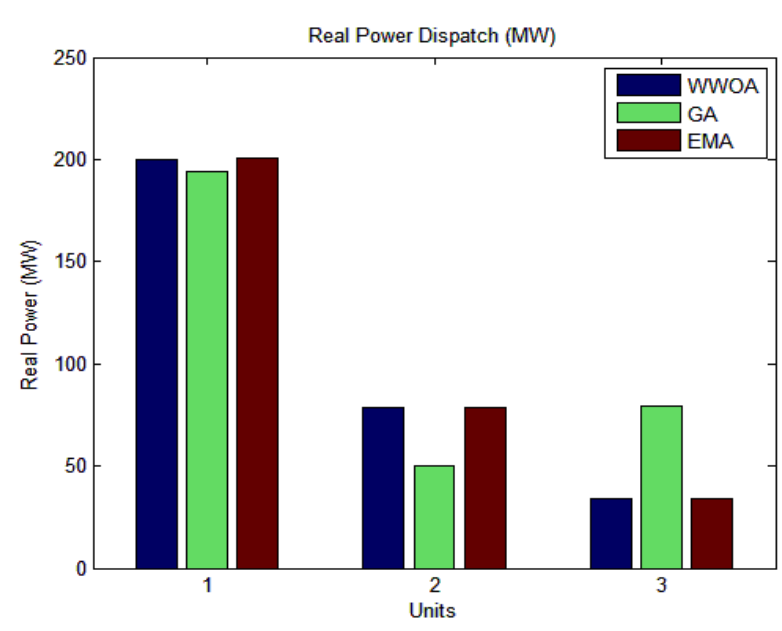

Figure.3 Generator outputs by WWOA compared with other algorithms for a three unit system

\subsection{Test system 2: 6 units system}

The test system has six thermal units and transmission losses are considered. The cost coefficients, minimum and maximum generation limits, ramp-rate limits and prohibited operating zones [18] of the generating units are given in table 1 . The system load demand is 1263 MW. Table 3 shows the revised operating regions after considering the prohibited operating zones and the ramp-rate constraints. The B-loss coefficients with base capacity of 100 MVA are used to calculate the transmission loss which is taken from [11]. The results obtained by solving the six unit test system are given in table 4 and its convergence characteristic is shown in figure 4 . The comparison of generator power outputs by WWOA with the algorithms compared is shown in figure 5 .

Table 3. Operating regions after considering the prohibited operating zones and ramp-rate constraints for a six unit system

\begin{tabular}{cccc}
\hline $\begin{array}{c}\text { Units } \\
\text { (MW) }\end{array}$ & $\begin{array}{c}\text { Operating } \\
\text { region 1 }\end{array}$ & $\begin{array}{c}\text { Operating } \\
\text { region 2 }\end{array}$ & $\begin{array}{c}\text { Operating } \\
\text { region 3 }\end{array}$ \\
\hline 1 & $(320-350)$ & $(380-500)$ & - \\
2 & $(80-90)$ & $(110-140)$ & $(160-200)$ \\
3 & $(100-150)$ & $(170-210)$ & $(240-265)$ \\
4 & $(60-80)$ & $(90-110)$ & $(120-150)$ \\
5 & $(110-140)$ & $(150-200)$ & - \\
6 & $(50-75)$ & $85-100)$ & $(105-120)$ \\
\hline
\end{tabular}

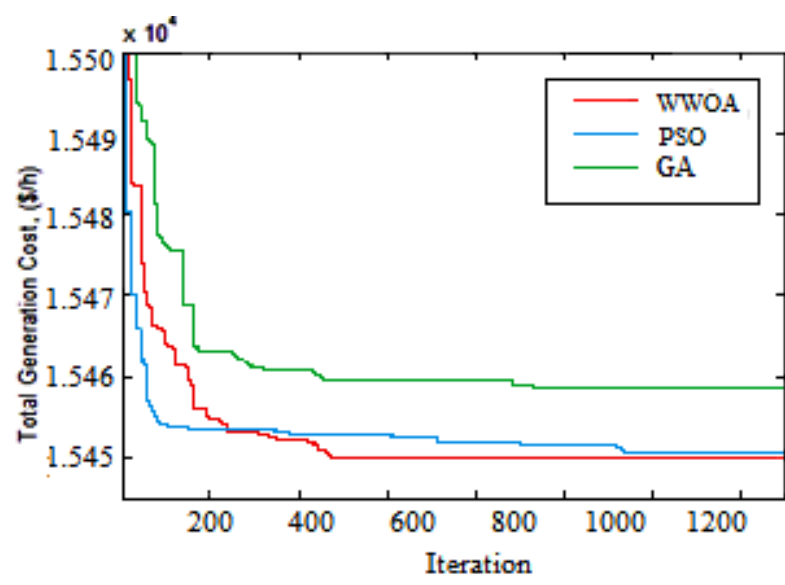

Figure.4 Convergence characteristics comparison of WWOA with other methods for a six unit system

The proposed algorithm without any roundup values of the computed output powers, produced the best solution as $15,449.8991(\$ / h)$. Figure 4 shows the comparison of convergence characteristics of proposed algorithm with PSO and GA techniques. From above figure, it is clear that WWOA finds better optimal solution than other algorithms considered. Also, the convergence speed of the proposed algorithm to reach the optimal solution is better when compared to other methods. The result of IDP method [7] is also compared in table 4 and since it is a mathematical approach, the convergence characteristic is not shown in the above figure.

The loss coefficients used from the references cited in the comparison table are four decimal places rounded up from the original loss coefficients.

\subsection{Test system 3: 15 units system}

In this fifteen-thermal units test system, the basic constraints and non-linear characteristics of the economic dispatch problem are implemented. The ramp-rate limits, prohibited operating zones and network transmission losses are also considered for demonstrating the proposed method and the test system's input data is taken from [18]. To ensure global optimum solution, a 50 different runs were performed by varying the total number of objective function evaluations. From the results it is observed that performance of the proposed approach is found to be better in terms of solution quality and convergence speed than the compared methods.

The input data for this system is provided in table 1. The load demand of the system is 2630 MW. The B-loss coefficient values for calculating the losses are taken from [11]. 
Table 4. Comparison of results for six units system (Pd=1263 MW)

\begin{tabular}{ccccc}
\hline Unit & $\begin{array}{c}\text { WWOA } \\
\text { (Proposed) }\end{array}$ & PSO [11] & IDP [7] & GA [11] \\
\hline P1 & 447.4982 & 447.4970 & 450.9555 & 474.8066 \\
P2 & 173.3397 & 173.3221 & 173.0184 & 178.6363 \\
P3 & 263.5061 & 263.4745 & 263.6370 & 262.2089 \\
P4 & 139.0724 & 139.0594 & 138.0655 & 134.2826 \\
P5 & 165.42 & 165.4761 & 164.9937 & 151.9039 \\
P6 & 87.12139 & 87.1280 & 85.3094 & 74.1812 \\
$\sum$ P & 1275.9576 & 1276.01 & 1275.98 & 1276.03 \\
PLss $_{\text {Loss }}$ & 12.9576 & 12.9584 & 12.9794 & 13.0217 \\
T. Gen. & 15449.8991 & 15450 & 15450 & 15459 \\
Cost $(\$ / h)$ & & & & \\
\hline
\end{tabular}

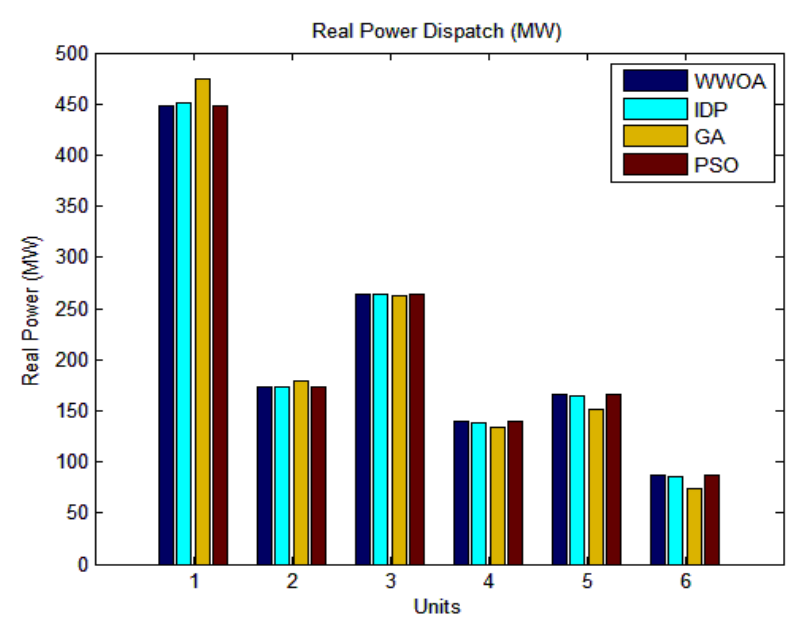

Figure.5 Generator outputs by WWOA compared with other algorithms for a six units system

Since the ramp-rate limits of the system restrict the operating range of the committed units between two operating periods, only the prohibited operating zone limits has to be considered. In this system, the units 2, 5, 6 and 12 have prohibited operating zones while the other nine units operate in their normal upper and lower limits. The regions of operation of these four units after including prohibited operating zone, ramp-rate limit constraints are given in table 5. The optimum generations of individual thermal units and the total fuel cost for a load demand of 2630 MW by the proposed algorithm and its comparison with other methodologies are reported in table 6 .

The convergence plot comparison and the comparison of generator power outputs of WWO Algorithm for a fifteen unit test system are shown in figure 6 and figure 7 respectively.

Table 5. Operating regions after considering the prohibited operating zone and ramp-rate constraints for a fifteen unit system

\begin{tabular}{cccc}
\hline Unit & $\begin{array}{c}\text { Operating } \\
\text { region 1 }\end{array}$ & $\begin{array}{c}\text { Operating } \\
\text { region 2 }\end{array}$ & $\begin{array}{c}\text { Operating } \\
\text { region 3 }\end{array}$ \\
\hline 2 & $(240-305)$ & $(335-420)$ & - \\
5 & $(150-180)$ & $(200-270)$ & - \\
6 & $(280-365)$ & $(395-430)$ & $(455-460)$ \\
12 & $(20-30)$ & $(40-55)$ & $(65-80)$ \\
\hline
\end{tabular}

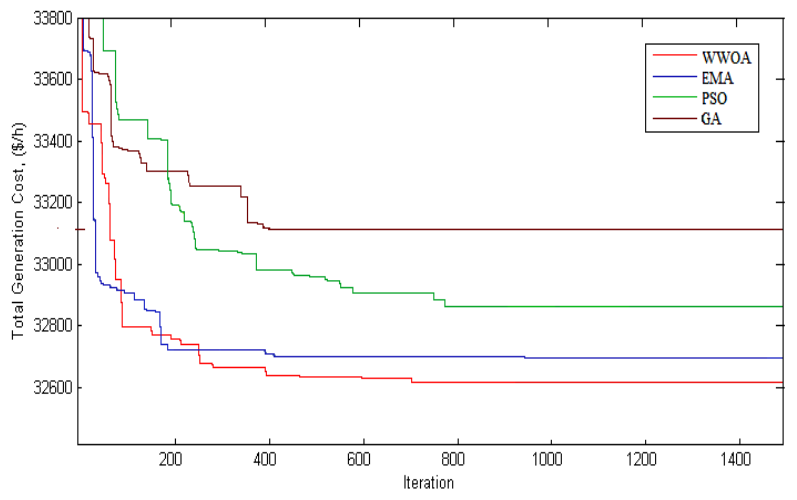

Figure.6 Convergence characteristics comparison of WWOA with other methods for a fifteen unit system 
$(\mathrm{Pd}=2630 \mathrm{MW})$

\begin{tabular}{ccccc}
\hline Unit & $\begin{array}{c}\text { WWOA } \\
\text { (Proposed) }\end{array}$ & EMA [18] & GA [11] & PSO [11] \\
\hline P1 & 455 & 455 & 415.3108 & 439.1162 \\
P2 & 440 & 380 & 359.7206 & 407.9727 \\
P3 & 130 & 130 & 104.4250 & 119.6324 \\
P4 & 13 & 130 & 74.9853 & 129.9925 \\
P5 & 267.64 & 170 & 380.2844 & 151.0681 \\
P6 & 460 & 460 & 426.7902 & 459.9978 \\
P7 & 430 & 430 & 341.3164 & 425.5601 \\
P8 & 60 & 72.0415 & 124.7867 & 98.5699 \\
P9 & 25 & 58.6212 & 133.1445 & 113.4936 \\
P10 & 46.0756 & 160 & 89.2567 & 101.1142 \\
P11 & 79.6989 & 80 & 60.0572 & 33.9116 \\
P12 & 79.6517 & 80 & 49.9998 & 79.9583 \\
P13 & 25 & 25 & 38.7713 & 25.0042 \\
P14 & 15 & 15 & 41.9425 & 41.4140 \\
P15 & 15 & 15 & 22.6445 & 35.6140 \\
$\sum P_{G i}$ & 2658.0662 & 2660.6626 & 2668.4 & 2662.4 \\
P 15 Loss & 28.0662 & 30.6626 & 38.2782 & 32.4306 \\
T. Gen. & 32616.4668 & 32704.4503 & 33113 & 32858 \\
Cost (\$/h) & & & & \\
\hline
\end{tabular}

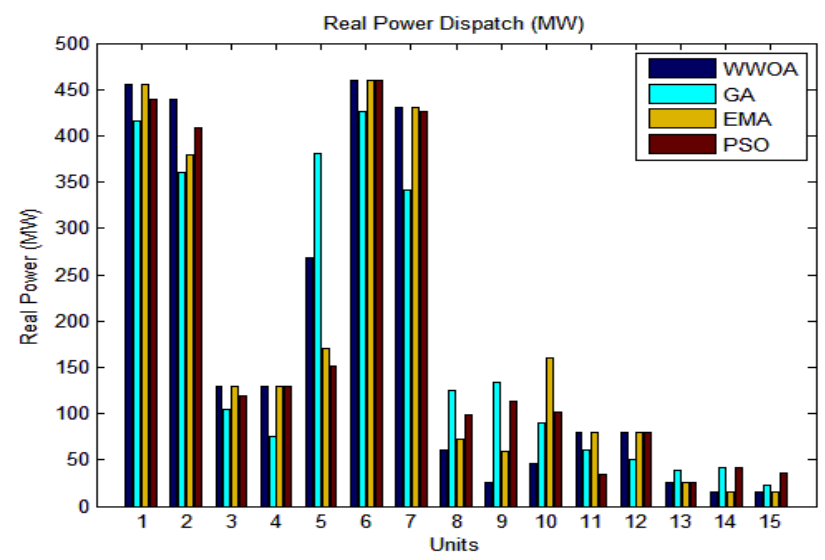

Figure.7 Generator outputs by WWOA compared with other algorithms for a fifteen unit system

\section{Conclusion}

In this paper, the economic dispatch problem of varying complexity has been considered and water wave optimization algorithm has been proposed to solve the problem. The problem has been subjected to various set of generator constraints. The analysis of the numerical simulation results renders the performance of the proposed algorithm for ELD problems. From the International Journal of Intelligent Engineering and Systems, Vol.9, No.4, 2016 results it is observed that WWOA can be considered as a reliable tool to solve ELD problems which have the ability to converge to a better quality solution. WWOA can also be implemented for solving complex hydrothermal scheduling, optimal power flow and dynamic economic load dispatch problems in power systems.

\section{Acknowledgements}

The authors thankfully acknowledge the authorities of Annamalai University, Annamalai Nagar, Tamil Nadu, India, for their sustained support and encouragement provided to conduct this research work.

\section{References}

[1] T.A.A. Victoire, A.E. Jeyakumar, "Hybrid PSO-SQP for Economic Dispatch with Valve-Point Effect", Electric Power System Research, Vol.71, No.1, pp.51-59, 2004.

[2] S. Kumar, R. Naresh, "Nonconvex Economic Load Dispatch Using an Efficient Real- Coded Genetic Algorithm", Applied Soft Computing, Vol.9, No.1, pp.321-329, 2009.

[3] W. M. Lin, F. S. Cheng and M. T. Tsay, "An Improved Tabu Search for Economic Dispatch with Multiple Minima," IEEE Trans. on Power Systems, Vol.17, pp.108-112, 2002.

[4] ZX. Liang, JD. Glover, "A Zoom Feature for a Dynamic Programming Solution to Economic Dispatch Including Transmission Losses", IEEE Trans. on Power Systems, Vol.7, No.2, pp.544-550, 1992.

[5] N. Sinha, R. Chakrabarti and P.K. Chattopadhyay, "Evolutionary Programming Techniques for Econ omic Load Dispatch", IEEE Trans. on Evolutionary Computation, Vol.7, No.1, pp.83-94, 2003.

[6] P. Somasundaram, K. Kuppusamy and D.R.P. Kumuudini, "Economic Dispatch with Prohibited Operating Zones using Fast Computation Evolutionary Algorithm", Electric Power Systems Research, Vol.70, No.3, pp.245-252, 2004.

[7] R. Balamurugan and S. Subramanian, "An Improved Dynamic Programming Approach to Economic Power Dispatch with Generator DOI: $10.22266 /$ ijies2016.1231.04 
Constraints and Transmission Losses", Journal of Electrical Engineering \& Technology, Vol.3, No.3, pp.320-330, 2008.

[8] N. Amjady and H. Sharifzadeh, "Solution of Nonconvex Economic Dispatch Problem Considering Valve Loading Effect by a New Modified Differential Evolution Algorithm", International Journal of Electrical Power \& Energy Systems, Vol.32, No.8, pp.893-903, 2010.

[9] A. Srinivasa Reddy, and K. Vaisakh, "Shuffled Differential Evolution for Economic Dispatch with Valve Point Loading Effects", International Journal of Electrical Power and Energy Systems, Vol.46, pp.342-352, 2013.

[10] J.B. Park, Y.W. Jeong and J.R. Shin and K.Y. Lee, "An Improved Particle Swarm Optimization for Non-convex Economic Dispatch Problems", IEEE Trans. on Power Systems, Vol.25, No.1, pp.156-166, 2010.

[11] Z.L. Gaing, "Particle Swarm Optimization to Solving the Economic Dispatch Considering the Generator Constraints", IEEE Trans. on Power Systems, Vol.18, No.3, pp.1187-1195, 2003.

[12] K.T. Chaturvedi, M. Pandit and L. Srivatsava, "Self-Organizing Hierarchical Particle Swarm Optimization for Nonconvex Economic Dispatch", IEEE Trans. on Power Systems, Vol.23, No.3, pp.1079-1087, 2008.

[13] A. Bhattacharya and P.K. Chattopadhyay, "Biogeography-Based Optimization for Different Economic Load Dispatch Problems", IEEE Trans. on Power Systems, Vol.25, No.2, pp.1064-1077, 2010.

[14] G. Xiong, D. Shi and X. Duan, "Multi-Strategy Ensemble Biogeography Based Optimization for Economic Dispatch Problems", Applied Energy, Vol. 111, pp. 801-811, 2013.

[15] S. Ozyon and D. Aydin, "Incremental Artificial Bee Colony with Local Search to Economic Dispatch Problem with Ramp Rate Limits and Prohibited Operating Zones", Energy Conversion and Management, Vol.65, pp.397-407, 2013.

[16] S. Hemamalini and S.P. Simon, "Artificial Bee Colony Algorithm for Economic Load Dispatch
Problem with Non-Smooth Cost Functions", Electric Power Components and Systems, Vol.38, No.7, pp.786-793, 2010.

[17] Y.J. Zheng, "Water Wave Optimization: A New Nature-Inspired Metaheuristic", Computers \& Operations Research, Vol.55, pp.1-11, 2015.

[18] N. Ghorbani and E. Babaei, "Exchange Market Algorithm for Economic Load Dispatch", International journal of Electrical power and Energy Systems, Vol.75, pp.19-27, 2016.

[19] P.H. Chen, C.H. Chang, "Large-Scale Economic Dispatch by Genetic Algorithm”, IEEE Trans. on Power Systems, Vol.10, no.4, pp.1919-1926, 1995.

[20] A.D. Craik, "The Origins of Water Wave Theory", Annual Review of Fluid Mechanics, Vol.36, No.1, pp.1-28, 2004.

[21] H. Huang, "Dynamics of Surface Waves in Coastal Waters: Wave-Current-Bottom Interactions", Higher Education Press, Beijing and Springer-Verlag, Berlin, 2009.

[22] V.E. Zakharov, V.S. L'vov, G. Falkovich, "Kolmo gorov Spectra of Turbulence I: Wave Turbulence”, Springer-Verlag, Berlin, 1992. 